\title{
Avoiding non-linearity of optically pumped magnetometer MEG within an actively shielded two-layer mu-metal room
}

\begin{abstract}
Some optically pumped magnetometer (OPM) sensors available for biomagnetic investigations have a linear range limited to $+-1 \mathrm{nT}$ due to the specific properties of their open loop operation. In a two-layer magnetically shielded room of type $\mathrm{Ak} 3 \mathrm{~b} / \mathrm{Vacoshield} \mathrm{Advanced} \mathrm{with} \mathrm{an} \mathrm{external}$ active compensation we studied how much sensor movement is allowed until amplitudes exceed the linearity range. Intentional movements were performed by a subject wearing an OPM-MEG sensor array. It was found that movements of 8 $\mathrm{cm}$ did yield non-linear amplitudes, but a reduction of the movement in half already preserves linearity. Despite movements, the heartbeat was found to generate a periodic signal, although the generating mechanism could not be identified so far.
\end{abstract}

Keywords: Magnetoencephalography, optically pumped magnetometer, non-linearity, movement artifact, heartbeat artifact

https://doi.org/10.1515/cdbme-2021-2138

\section{Introduction}

The magnetic signature of brain activity measured by magnetoencephalography (MEG) has found new interest through the availability of optically pumped magnetometers (OPM). They do not require a fixed geometry cryogenic Dewar as it is the case with superconducting quantum interference devices (SQUID) used traditionally for MEG. One disadvantage of current OPMs is the requirement to have background fields of less than $1 \mathrm{nT}$ for linear operation [1]. To achieve this in a typical magnetically shielded room (MSR) great progress was made in [2] using a compensation coil

\footnotetext{
${ }^{*}$ Corresponding author: Tilmann H. Sander: PhysikalischTechnische Bundesanstalt, Berlin, Germany, tilmann.sanderthoemmes@ptb.de

Urban Marhl: University of Maribor, Maribor, Slovenia

Vojko Jazbinšek: Institute of Mathematics, Physics and

Mechanics, Ljubljana, Slovenia
}

system originally developed for magnetic resonance imaging. If the movement of subjects is not required as in [2] a tripleaxis compensation on the outside surfaces of an Ak3b (vacuumschmelze.com) [3] reduces the fluctuating fields sufficiently to record event related fields such as from hearing tones [4].

Here it will be investigated how much head movement is allowed in the simple configuration described in [3] and if other sources of noise interact with the field gradient in an MSR.

\section{OPM-MEG using helmet-type} sensor holders

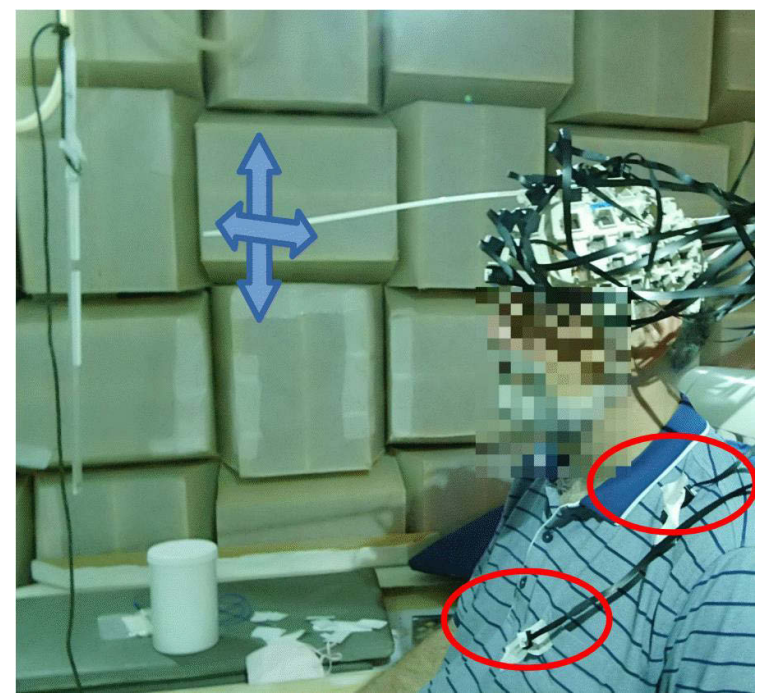

Figure 1: Subject wearing the OPM-MEG helmet and two additional OPMs placed on thorax and shoulder (red circles). The subject views a ruler cross and with the stick attached to the helmet the subject sees the range of the self-initiated movements. The blue arrows indicate the two movement directions studied, left/right, and up/down.

The experimental setup consists of an array of 25 dual-axis sensors of type QZFM Gen 2.0 made by QuSpin (quspin.com). The sensors are inserted into a 3D printed helmet based on the 
individual head shape of the subject. In Fig. 1 the subject is wearing the sensor helmet for the OPM-MEG and is seated comfortably inside an MSR.

Since the OPM-MEG sensors are rigidly attached to the subject's head any movement by the subject generates a signal in the OPMs due to the field gradients in the MSR. This contrasts with SQUID-MEG where only vibrations of the stationary Dewar induce signals of this type. Usually, the subject is instructed to stay still and perform the given task such as in [4]. Here, as shown in Fig. 1 the OPM-MEG sensor holder has a protruding stick, which allows the subject to observe the head movement distance by observing the stick in relation to crossed rulers suspended from the ceiling. Two additional OPM sensors were attached on the thorax and shoulder to record the biomagnetic signal of the heart, the magnetocardiogram (MCG), which is known to be a source of noise in SQUID-MEG [5].

\section{OPM signals during movement}

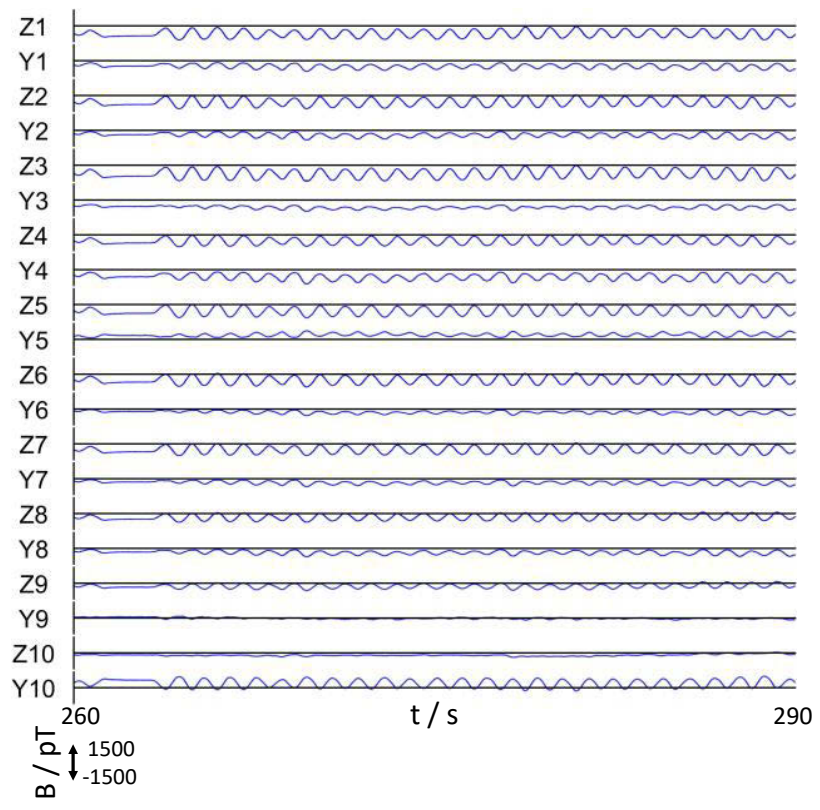

Figure 2: Magnetic field measured by representative OPM-MEG sensors during $30 \mathrm{~s}$ of left/right movements of $8 \mathrm{~cm}$ peak-topeak travel. The signals reach up to $1.5 \mathrm{nT}$ exceeding the linearity threshold of the OPMs of $1 \mathrm{nT}$.

The experiment consisted of an initial $200 \mathrm{~s}$ of rest, where the subject was instructed to keep eyes open and to sit comfortably still. Then the outside operator instructed the subject to perform left/right head movements of 2,4 , and $8 \mathrm{~cm}$ extent, each for $30 \mathrm{~s}$. After the sideways movements up/down movements of the same extent were performed.
The signals resulting from the $8 \mathrm{~cm}$ left/right movements are shown in Fig. 2. The Zx-label denotes the intrinsic Zsensing and the Yx-label the intrinsic $\mathrm{Y}$-sensing direction of each dual-axis OPM sensor, which are not related to the $\mathrm{Z}$ - and Y-directions of the MSR. The sensors are distributed over the helmet shaped sensor holder and therefore the signal amplitude induced by the movement can vary between sensors and between the Z- and Y-direction of each sensor, e.g. Z4 and Y4 have a similar amplitude, but Z10 and Y10 greatly differ. Furthermore, the magnetic gradient field in the MSR is not known in detail and therefore the local gradient direction projected onto the sensor direction determines the induced amplitude.

Important is that the signals in Fig. 2 have the same phase, suggesting a common origin of the oscillations. The oscillation amplitude reaches up to $1.5 \mathrm{nT}$. The gradient in the MSR is roughly $20 \mathrm{nT} / \mathrm{m}$ and therefore the value of $1.5 \mathrm{nT}$ is consistent with a movement distance of $8 \mathrm{~cm}$, since $20 \mathrm{nT} *$ $0.08=1.6 \mathrm{nT}$.

To quantify the sensor amplitude behavior the signal distribution functions have been calculated and are shown in Fig. 3 (A) for $30 \mathrm{~s}$ of rest data and (B) for the $30 \mathrm{~s}$ of movement data from Fig. 2. The rest data are within the linearity limits of $+-1 \mathrm{nT}$ indicated by the red vertical lines. The center of the distribution varies between sensors as the field locking mechanism of the sensors depends on local field variations and can be an individual intrinsic sensor property. For comparative reasons the rest distribution is calculated from $30 \mathrm{~s}$ of data, but it was tested to be stable for the full $200 \mathrm{~s}$ of rest data recorded.

In contrast, the $8 \mathrm{~cm}$-movement induced signals for several sensors clearly exceed the linearity range of $1 \mathrm{nT}$ as the distribution extends across the red vertical lines in Fig. 3 (B). Inspecting the signals of the $4 \mathrm{~cm}$ movements (not shown) linear behavior of the OPMs was guaranteed and the up/down movements did give similar results. Note that the better gradient suppression described in [2] allows complex movements of up to $10 \mathrm{~cm}$ distance without exceeding the linearity range of the OPMs.

\section{Heartbeat related signals}

Intrinsic physiological artifact sources such as eye blinks or heart activity have always been important in MEG since they can have amplitudes exceeding the brain signals of interest. Eye blinks can be controlled by a subject to a certain degree, but the magnetic signals originating from the heart muscle are always present. A stimulation sequence such as, e.g., the presentation of tones is usually chosen to be uncorrelated to the heartbeat. Nevertheless, the amplitude of heartbeat related 

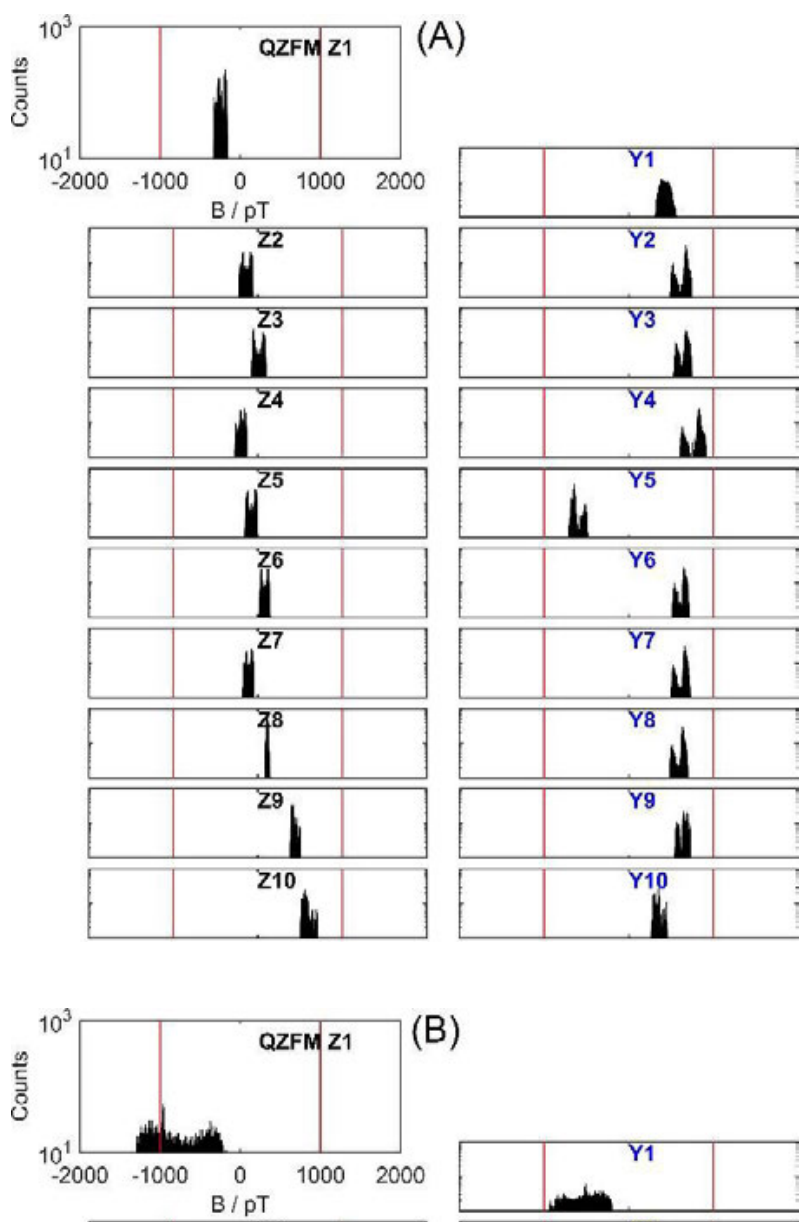

(B)
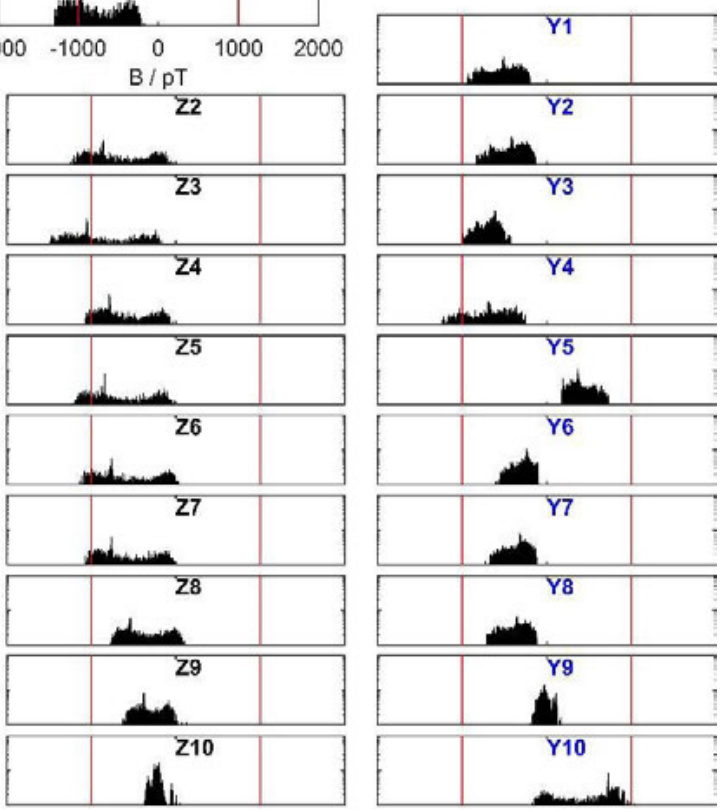

Figure 3: (A) Signal distribution function of representative OPMMEG sensors with the subject at rest. All signals are in the linearity range of $+-1 \mathrm{nT}$ indicated by the red lines. (B) Signal distribution function of the OPM-MEG sensors with the subject performing left/right movements of $8 \mathrm{~cm}$ peak-to-peak travel. Some signals exceed the linearity range. signals in the MEG sensors needs to be known to estimate its influence. To investigate this two OPM sensors were placed over the thorax and shoulder as shown in Fig. 1. The signal from these sensors, Z19 and Z20, can be seen in Fig. 4 at the bottom for a section of $10 \mathrm{~s}$ of rest data. Both show clearly the MCG, Z19 shows a strong R-peak and Z20 shows a small Rpeak and a slow wave with a periodicity of $\sim 3 \mathrm{~s}$. The slow wave is likely to be due to the respiratory movement of the shoulder. Since this slow wave is visible only in Z20 and not in Z19 and not in the OPM-MEG sensors Z10/Y10 and Z11/Y11 the respiratory artifact signal seems to be localized.

In contrast to MEG measured with SQUIDs [5], the MCG is not directly visible in the OPM-MEG signals shown here. The signals Z10/Y10 and Z11/Y11 are representative for all OPM-MEG signals, i.e., a heartbeat related peak was not observed in any of the signals of the 25 OPM sensors. It might just be that the heart muscle signal is obscured by the higher noise of OPM sensors $(10-20 \mathrm{fT} / \mathrm{sqrt}(\mathrm{Hz}))$ compared to SQUID sensors (3-8 fT/sqrt(Hz)). Therefore, the averaged MCG was calculated for the OPM-MEG signals using the Rpeak times as a trigger for averaging of the data. The resulting averaged MCG is shown in Fig. 5 for the same signals which were included in Fig. 4. Note the scaling for Z19 and Z20, which means the R-peak over the thorax has an amplitude of $60 \mathrm{pT}$, which agrees with other studies [6]. In the averaged OPM-MEG signals Z10/Y10 and Z11/Y11 a wave-like pattern is observed, but not a sharp peak like the R-peak. Since the wave-like pattern seems to be strongly correlated across OPMMEG signals it is likely to be related to the heartbeat.

With an amplitude of up to $10 \mathrm{pT}$ the wave-like pattern might have an influence on averaged OPM-MEG signals, but further research is needed to understand how the wave like pattern is generated. A conjecture is that the pulsation of the blood generates a very small movement of the OPM-MEG sensor helmet and this induces a signal through the movement of the OPM-MEG in the gradient of the MSR. Taking the amplitude of $10 \mathrm{pT}$ observed in Fig. 5 a movement of $0.5 \mathrm{~mm}$ can be estimated (cf. above, gradient estimate is $20 \mathrm{nT} / \mathrm{m}$ ) and this is not unreasonable for a person sitting upright in a chair. Clearly, independent movement measurements would be required to support this hypothesis.

\section{Conclusions}

Non-linear signals were induced in OPMs attached to a subject's head by sideway head movements of $8 \mathrm{~cm}$. Head movements of $4 \mathrm{~cm}$ distance yield already linear behavior. Gradient compensation schemes such as demonstrated in [2] allow to study cognitive paradigms involving movements as 
part of the task. Here it is demonstrated that a simpler setup with an external active compensation allows to harness the advantages of OPMs for MEG if the subject is seated and instructed not to move. Future work should clarify the origin of the heartbeat related artifact and aim for its reduction.

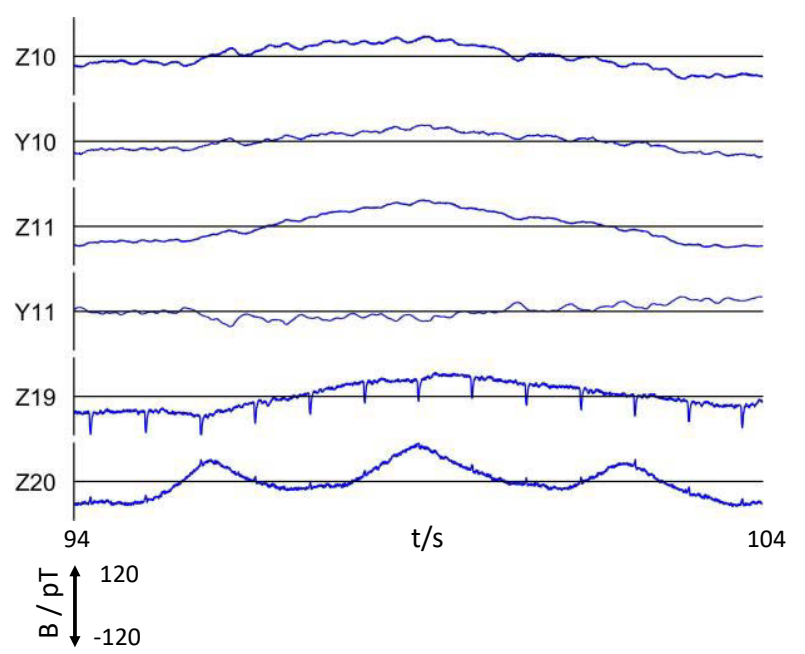

Figure 4: Signals of the OPMs attached to thorax (Z19) and shoulder (Z20) together with representative OPM-MEG sensors. The heartbeat is clearly visible in Z19. Signal Z20 shows a fluctuation most likely related to respiration. The OPM-MEG signals do not show any obvious heartbeat or respiration related contributions.

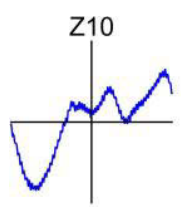

Z19
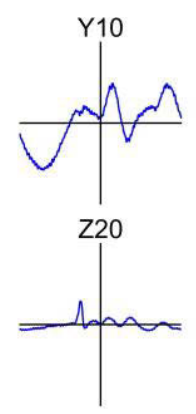
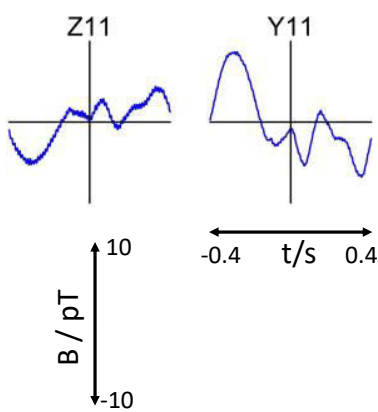

$0.4 \quad \mathrm{t} / \mathrm{s} \quad 0.4$

Figure 5: Signals of the OPMs from Fig. 4 averaged using the Rpeak of the heart cycle as trigger. The R-peak is clearly visible in Z19 and weaker in Z20 (Note that the signals of Z19 and $Z 20$ have been reduced by a factor of 6 here for visualization). The OPM-MEG sensors do not show the Rpeak, but a wave-like activity clearly related to the heartbeat. The wave-like activity is similar in the four OPM-MEG signals shown here and it is unlikely to be related to individual sensor noise as it has an amplitude of $10 \mathrm{pT}$. A similar signal pattern is observed in the other OPM-MEG sensors.

\section{Author Statement}

Support by DAAD project ID 57402032 and Slovenian Research Agency project ID P2-0348 is acknowledged. Help by Katrijn Everaert is gratefully acknowledged. Authors state no conflict of interest. The research was performed in accordance with the tenets of the Helsinki Declaration and informed consent has been obtained from all individuals. The study has been approved by the institutional review board of PTB.

\section{References}

[1] Osborne J, Orton J, Alem O, Shah V (2018) Fully integrated, standalone zero field opticall pumped magnetometer for biomagnetism. Proc SPIE 10548, Steep Dispersion Engineering and Opto-Atomic Precision Metrology XI:1-7.

[2] Holmes N, Leggett J, Boto E, Roberts G, Hill RM, Tierney TM, Shah V, Barnes GR, Brookes MJ, Bowtell R (2018) A biplanar coil system for nulling background magnetic fields in scalp mounted magnetoencephalography. Neuroimage 181:760-774.

[3] Sander TH, Marhl U, Brühl R, Middelmann T, Jazbinšek V (2021) A 50 channel optically pumped magnetometer MEG in an externally actively shielded two-layer room. Intl. J. Bioelectromagnetism, in press.

[4] Marhl U, Jodko-Wladzinsk A, Brühl R, Sander TH, Jazbinšek V (2021) Comparison between conventional SQUID based and novel OPM based measuring systems in MEG. In T. Jarm et al. (Eds.): EMBEC 2020, IFMBE Proc 80:254-261.

[5] Sander TH, Burghoff M, Van Leeuwen P, Trahms L (2007) Application of decorrelation-independent component analysis to biomagnetic multi-channel measurements. Biomed Tech (Berl.) 52:130-6.

[6] Alem O, Mhaskar R, Jiménez-Martínez R, Sheng D, LeBlanc J, Trahms L, Sander T, Kitching J, Knappe S (2017) Magnetic field imaging with microfabricated optically-pumped magnetometers. Opt Express 25:7849-7858. 